\title{
A survey on the provision of dental care for children with cleft lip and palate
}

\author{
Provision of general dental care for children with cleft lip and palate - parental attitudes and experiences \\ by S. Mc Donagh, R. Pinson and A. J. Shaw Br Dent J 2000; 189: 432-434
}

\author{
Objective \\ To evaluate the attitudes of parents of 4-8 year-old children with \\ cleft lip and palate (CLP) towards the provision of paediatric \\ dental care and to assess their experience of treatment within the \\ General Dental Services.

\section{Design} \\ Postal questionnaire distributed to all parents of 4-8 year-old \\ children on the Birmingham CLP database.

\section{Results} \\ The response rate was $77 \%$. Ninety-nine $(91 \%)$ children were \\ registered with a dentist. Seventy-five $(69 \%)$ had previously \\ received preventive advice and $32(29 \%)$ had experienced \\ restorative intervention. The majority of parents $(64 \%)$ \\ expressed a wish for a dental check-up to be provided at the \\ designated Cleft Centre, with 42 (39\%) requesting preventive \\ advice. Fifty-eight $(67 \%)$ of the parents who requested a dental \\ check-up were agreeable for treatment to be provided in the \\ primary sector.
}

\author{
Conclusion \\ The survey indicates there is parental support for paediatric \\ dental assessment at cleft clinics with subsequent arrangement of \\ treatment in the primary sector. The inclusion of paediatric \\ dental support within the multidisciplinary cleft team should be \\ considered as Regional Cleft Centres are established.
}

In Brief

- This paper establishes the desire of parents of children with CLP for preventive advice to be made available at cleft centres.

- The potential benefits of paediatric dental input centrally are highlighted.

- The need for consideration of preventive services as the centralized cleft centres are established is discussed.

\section{Comment}

The Clinical Standards Advisory Group 1 (CSAG) survey of cleft lip and palate patient care in the United Kingdom reviewed the dental health of the cleft lip and palate patients in their study, and found poor dental outcomes. This is therefore a timely study, looking, as it does, at the actual provision of dental care for children with cleft lip and palate in the Birmingham area. The study is well designed, with care being taken to compile the questionnaire, taking account of the ethnic language variation within Birmingham. The age range of 4 to 8 years old seems well chosen as it represents the period of early dental contact, which sets the scene for future dental care.

The percentage response of the questionnaires $(77 \%)$ seems good, and it was pleasing to see $91 \%$ of these children were registered with a general dental practitioner. One wonders what percentage of
\end{abstract}

the $23 \%$ of non-respondents would be registered? There was also an impressively high number of those registered who were seen for check-up in the preceding year (91\%).

With the variation in dental status so varied throughout the United Kingdom, and varied even within cities, the Birmingham experience of dental treatment in a fluoridated area needs to be interpreted with care. It is not clear how many of the children had seen a paediatric dentist before, but $64 \%$ of parents would like a dental check-up to take place at a cleft centre. Nearly half (46\%) of these parents however, wished the necessary treatment to be carried out by their own general dental practitioner, closer to home, which is practical and sensible.

The central visit to the hub/ cleft centre is important to build up a comprehensive picture of care by taking measurements, which sets the baseline for dental care in the area covered by the cleft team.

In the discussion in the paper, the importance of giving comprehensive dental health advice is stressed. Whereas $91 \%$ of children are registered with dentists, a possible vulnerable group exists within this percentage, and of the $23 \%$ who do not respond.

The 'central cleft team' check is therefore important, not solely as a record of the level of care, but also as an important means of identifying the children at risk dentally, and providing appropriate dental treatment and support. A strong case is therefore made for the inclusion of paediatric dental input to the core cleft team at the hub of the newly established cleft centres.

\section{Iain S. Hathorn}

Consultant Orthodontist, United Bristol Healthcare Trust 